\title{
LATTICE NORMALITY AND OUTER MEASURES
}

\section{PANTAGIOTIS D. STRATIGOS}

\author{
Department of Mathematics \\ Long Island University \\ Brooklyn, NY 11201
}

(Received August 20, 1991 and in revised form March 26, 1992)

ABSTRACT. A lattice space is defined to be an ordered pair whose first component is an arbitrary set $x$ and whose second component is an arbitrary lattice $L$ of subsets of $x$. A lattice space is a generalization of a topological space. The concept of lattice normality plays an important role in the study of lattice spaces.

The present work establishes various relationships between normality of lattices of subsets of $x$ and certain "outer measures" induced by measures associated with the algebras of subsets of $x$ generated by these lattices.

KEY WORDS AND PHRASES. Lattice space, lattice normality, lattice regularity and o-smoothness of a measure, weak lattice regularity of a measure, finitely subadditive outer measure; countably subadditive outer measure.

1991 AMS SUBJECT CLASSIFICATION CODES. 28A12, 28A60, 28C15.

1. INTRODUCTION.

It is our aim in this paper to establish various relationships between normality of lattices on an arbitrary set and certain "outer measures" induced by measures on the algebras generated by these lattices.

More specifically, consider any set $x$ and any lattice on' $x, L$. The algebra on $x$ generated by $L$ is denoted by $A(L)$.

In Section 3, a finitely subadditive "outer measure" is associated with an arbitrary (0-1)-valued measure on $A(L)$. The behavior of "outer measures" of this type on $L$ can be used effectively to characterize normal lattices, and this is investigated in section 3. Also in Section 3, a notion of weak regularity of measures is introduced in terms of the associated finitely subadditive "outer measures" and it is shown that if $L$ is normal, then this notion coincides with regularity.

In Section 4, a countably subadditive "outer measure" is associated with an arbitrary $(0-1)$-valued measure on $A(L)$. The relationship between this "outer measure" and the associated (0-1)-valued measure is considered in the presence of smoothness. Also in Section 4, the equality of certain of these mcasures and "outer measures" on $L$ or $L^{\prime}$ - the complementary lattice of $L$ - is considered, in particular in the case where $L$ is normal and countably paracompact or $L$ is 
normal and $\delta$. In addition, conditions on $L$ are given, including normality, which automatically imply regularity of certain smooth measures.

Typical applications are given throughout the paper in the case of topological lattices; it is clear that many more such applications could be given. It is also clear that certain of our results extend to arbitrary measures, but we will pursue this matter elsewhere.

We adhere to standard by now lattice terminology and notation, which can be found, for example, in $[1,2,6,7]$ and, for convenience, we review some of the more important terminology and notation used throughout the paper.

2. TERMINOLOGY AND NOTATION.

(a) Consider any set $x$ and any lattice on $x, L$. We shall assume that $\varnothing, x \in L$, without loss of generality for our purposes.

Now, consider any topological space $x$ and denote the class of open sets by $U$, the class of closed sets by $F$, the class of clopen sets by $C$, and the class of zero sets by $Z$. Note each of the classes $U, F, C, Z$ is a lattice of the prescribed type. Recall $U$ is also referred to as the topology on $x$ and the topological space $x$ is defined to be $(x, U)$. Thus $(x, L)$ is a generalization of a topological space. For this reason, we shall refer to $\langle x, L\rangle$ as a lattice space. In topological measure theory, it is convenient to regard $F$ as the topology on $x$ and $(x, F\rangle$ as the topological space.

$L$ is said to be complement generated iff for every element of $L$, $L$, there exists a sequence in $L,\left\langle\tilde{L}_{n}\right\rangle$, such that $L=n_{n} \tilde{L}_{n}^{\prime} \cdot L$ is said to be normal iff for every two elements of $L, A, B$, if $A \cap B=\varnothing$, then there exist two elements of $L, C, D$, such that $A \subset C^{\prime}$ and $B \subset D^{\prime}$ and $C^{\prime} \cap D^{\prime}=\varnothing . \quad L$ is said to be countably paracompact iff for every sequence in $L,\left(A_{n}\right)$, if $\left(A_{n}\right)$ is decreasing and $\lim _{n} A_{n}=\varnothing$, then there exists a sequence in $L,\left\langle B_{n}\right\rangle$, such that for every $n, A_{n} \subset B_{n}^{\prime}$ and $\left\langle B_{n}^{\prime}\right\rangle$ is decreasing and $\lim _{n} B_{n}^{\prime}=\varnothing$.

(b) The algebra on $x$ generated by $L$ is denoted by $A(L)$. Consider any algebra on $X, A$. A measure on $A$ is defined to be a function $\mu$ from $A$ to $R$ such that $\mu$ is finitely additive and bounded. (See [1], p. 567.) The set whose general element is a measure on $A(L)$ is denoted by $M(L)$. An element of $M(L), \mu$, is said to be $L$-regular iff for every element of $A(L), E$, for every positive number $\varepsilon$, there exists an element of $L, L$, such that $L \subset E$ and $|\mu(E)-\mu(L)|<\varepsilon$. The set whose general element is an element of $M(L)$ which is $L$-regular is denoted by $M_{R}(L)$. An element of $M(L), \mu$, is said to be $L-(\sigma-s m o o t h)$ iff for every sequence in $A(L)$, $\left\langle A_{n}\right\rangle$, if $\left\langle A_{n}\right\rangle$ is decreasing and $\lim _{n} A_{n}=\varnothing$, then $\lim _{n} \mu\left(A_{n}\right)=0$. The set whose general element is an element of $M(L)$ which is $L-(\sigma-s m o o t h)$ is denoted by $M^{\sigma}(L)$. The set whose general element is an element of $M(L)$ which is $L-(\sigma-s m o o t h)$ just for ' $A_{n}$ ' in $L$ is denoted by $M_{\sigma}(L)$.

The set whose general element is an element of $M(L), \mu$, such that $\mu(A(L))=\{0,1\}$, that is, the set whose general element is a $(0-1)$-valued measure on $A(L)$ is denoted by $I(L)$.

NOTE. Since every element of $M(L)$ is expressible as the difference of nonnegative elements of $M(L)$, we shall work with nonnegative elements of $M(L)$, without loss of generality. (Related matters can be found, for example, in $[3,4,5,8]$. ) 
(c) A finitely subadditive outer measure is defined to be a function $\phi$ from $P(x)$ to $R$ such that $\phi(\varnothing)=0$ and $\phi$ is nonnegative, increasing, and finitely subadditive.

A countably subadditive outer measure is defined to be a function $\phi$ from $P(x)$ to $R$ such that $\phi(\varnothing)=0$ and $\phi$ is nonnegative, increasing, and countably subadditive. 3. LATTICE NORMALITY AND FINITELY SUBADDI'TIVE OUTER MEASURE.

In this section, we work with an arbitrary set $X$ and an arbitrary lattice on $x, L$. We introduce a certain finitely subadditive outer measure on $P(x)$ and use it to obtain conditions for $L$ to be normal.

DEFINITION 3.1. Consider any lattice space $(x, L)$. Nọw, consider any element of $M(L), \mu$, and the function $\mu^{\prime}$ on $P(X)$ determined by $\mu^{\prime}(A)=\inf \left\{\mu\left(L^{\prime}\right) \mid L \in L\right.$ and L' $\supset$ A\}.

PROPOSITION 3.2. (i) $\mu^{\prime}$ is a finitely subadditive outer measure.

(ii) $\mu=\mu^{\prime}$ on $L^{\prime}$.

(iii) $\mu \leq \mu^{\prime}$ and $\mu=\mu^{\prime}$ iff $\mu$ is L-regular.

(iv) If $\mu \in I(L)$, then $\mu^{\prime}(P(X))=\{0,1\}$.

(Proof omitted.)

The following theorem gives a characterization of normality of $L$ in terms of finitely subadditive outer measures of the type introduced by the preceding definition.

LEMMA 3.3. $L$ is normal iff for every element of $I(L), \mu$, for every two elements of $I_{R}(L), v_{1}, v_{2}$, such that $\mu \leq v_{1}, v_{2}$ on $L_{1} v_{1}=v_{2}$, (Known.)

THEOREM 3.4. The following statements are equivalent:

(a) $L$ is normal.

(b) For every element of $I_{R}\left(L^{\prime}\right) \mu$, for every element of $I_{R}(L), v$, such that $\mu \leq \nu$ on $L, \mu^{\prime}=v^{\prime}$ on $L$.

(c) For every $\mu$ and $\nu$, as in (b), for every element of $L, A$, such that $v\left(A^{\prime}\right)=1$, there exists an element of $L, B$, such that $B \subset A^{\prime}$ and $\mu(B)=1$.

PROOF. (a) implies (b). Assume (a) and show (b). Consider any element of $I_{R}\left(L^{\prime}\right), \mu$, and any element of $I_{R}(L), \nu$, such that $\mu \leq \nu$ on $L$ and show $\mu^{\prime}=\nu^{\prime}$ on

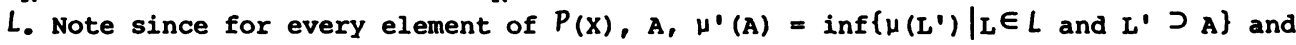
$\nu^{\prime}(A)=\inf \left\{\nu\left(L^{\prime}\right) \mid L \in L\right.$ and $\left.L^{\prime} \supset A\right\}$ by definition and $\nu \leq \mu$ on $L^{\prime}$ because $\mu \leq \nu$ on $L$ by assimption, $v^{\prime} \leq \mu^{\prime}$. Hence to show $\mu^{\prime}=V^{\prime}$ on $L^{\prime}$, it suffices to show for every element of $L, A, V^{\prime}(A) \ngtr \mu^{\prime}(A)$. Assume the contrary. Then there exists an element of $L, A$, such that $\nu^{\prime}(A)<\mu^{\prime}(A)$. Consider any such $A$. Then since $\mu, \nu \in I(L), V^{\prime}(A)=0$ and $\mu^{\prime}(A)=1$. Now, note since $\nu$ is $L$-regular by assumption, $v=v^{\prime}$. Consequently $v(A)=0$. Hence since $\nu \in I_{R}(L)$, there exists an element of $L, L$, such that $L^{\prime} \supset A$ and $\nu\left(L^{\prime}\right)=0$. Consider any such $L$. Then since $L$ is normal by assumption, there exist two elements of $L, C, D$, such that $A \subset C^{\prime} \subset D^{\prime} \subset L^{\prime}$. Consider any such $C_{\text {, }} D$. Then $\mu^{\prime}\left(C^{\prime}\right) \leq \mu(D) \leq \nu(D) \leq \nu\left(L{ }^{\prime}\right)=0$. IIence $\|\left(C^{\prime}\right)=0$. Consequently $\mu^{\prime}(A)=0$. Thus a contradiction has been reached. Therefore, the assumption is wrong. Consequently $\mu^{\prime}=V^{\prime}$ on $L^{\text {. }}$ (Note the $L^{\prime}$-regularity of $\mu$ was not needed in the proof.)

(b) implies (a). Assume (b) and show (a). For this, use Lemma 3.3, namely, 
consider any element of $I(L), \rho$, and any two elements of $I_{R}(L), v_{1}, v_{2}$, such that $\rho \leq \nu_{1}, v_{2}$ on $L$ and show $v_{1}=v_{2}$. Note since $\rho \in I(L)\left(=I\left(L^{\prime}\right)\right)$ by assumption, there exists an element of $I_{R}\left(L^{\prime}\right), \mu$, such that $\rho \leq \mu$ on $L^{\prime} \cdot$ Consider any such $\mu$. Then $\mu \leq \rho$ on $L$. Hence since $\rho \leq \nu_{1}, \nu_{2}$ on $L$ by assumption, $\mu \leq \nu_{1}, v_{2}$ on $L$. Thus $\mu \in I_{R}\left(L^{\prime}\right)$ and $v_{1}, v_{2} \in I_{R}(L)$ and $\mu \leq \nu_{1}, v_{2}$ on $L$. Hence since $(b)$ is true by

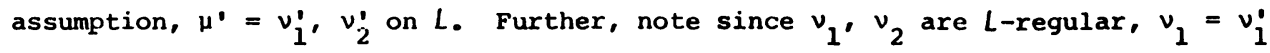
and $v_{2}=v_{2}^{\prime}$. Consequently, $v_{1}=v_{2}$. Then by Lemma $3.3, L$ is normal.

(b) is equivalent to (c). [Proof omitted. (Again it should be noted that the L'-regularity of $\mu$ is not needed in the proof of (b) implies (c).)]

APPLICATION 3.5. Consider any topological space $x$. Then according to Theorem 3.4, the following statements are equivalent: (a) $X$ is normal. (b) For every element of $I_{R}(U), \mu$, for every element of $I_{R}(F), \nu$, such that $\mu \leq \nu$ on $F, \mu^{\prime}=\nu^{\prime}$ on $F$. (c) For every $\mu$ and $v$, as in (b), for every element of $U, U$ such that $\nu(U)=1$, there exists an element of $\Gamma, F$, such that $F \cup U$ and $\mu(F)=1$.

APPLICATION 3.6. Consider any topological space $X$ such that $X$ is $T_{3 \frac{1}{2}}$ Then since $Z$ is normal, according to Theorem 3.4, the following statements are true: (b) For every element of $I(Z), \mu$, for every element of $I_{R}(Z), v$, such that $\mu \leq v$ on $Z, \mu^{\prime}=v^{\prime}$ on $Z$. (c) For every $\mu^{\prime}$ and $v^{\prime}$, as in (b), for every element of $Z, Z$, such that $\nu\left(z^{\prime}\right)=1$, there exists an element of $Z, \tilde{z}$, such that $\tilde{z} \subset z^{\prime}$ and $\mu(\tilde{z})=1$.

THEOREM 3.7. If $L$ is normal, then for every element of $I_{\sigma}(L)$, $\mu$, for every element of $I_{R}(L), \nu$, such that $\mu \leq \nu$ on $L, \nu \in I_{\sigma}\left(L^{\prime}\right)$.

PROOF. Assume $L$ is normal. Consider any element of $I_{\sigma}(L), \mu$, and any element of $I_{R}(L), \nu$, such that $\mu \leq \nu$ on $L$. To show $\nu \in I_{\sigma}(L)$, assume the contrary. Then by the relevant definition, there exists a sequence in $L_{,}\left\langle L_{n}\right\rangle$, such that $\left\langle L_{n}^{\prime}\right\rangle$ is decreasing and $\lim _{n} L_{n}^{\prime}=\varnothing$ and $\lim _{n} \nu\left(L_{n}^{\prime}\right) \neq 0$. Consider any such $\left(L_{n}\right)^{\prime}$. Then since $\nu \in I(L)$ by assumption, for every $n, v\left(L_{n}^{\prime}\right)=1$. Hence for every $n$, since $\mu \in I\left(L^{\prime}\right)$ and $\nu \in I_{R}(L)$ and $\mu \leq \nu$ on $L$ and $L$ is normal by assumption, by Theorem 3.4, there exists an element of $L, \hat{I}_{n^{\prime}}$ suchl that $\hat{r}_{n} \subset I_{n}^{\prime}$ and $\|\left(\hat{L}_{n}\right)=1$; considicr any suchl $\hat{L}_{n}$. Now, for every $n$, consider $n_{k=1}^{n} \hat{L}_{k} ;$ note $n_{k=1}^{n} \hat{L}_{k} \in L_{i} \operatorname{set} n_{k=1}^{n} \hat{L}_{k}=\tilde{L}_{n}$. Further, consider $\left\langle\tilde{L}_{n}\right\rangle$. Note $\left\langle\tilde{L}_{n}\right\rangle$ is in $L$ and for every $n, \mu\left(\tilde{L}_{n}\right)=1$ and $\tilde{L}_{n} \subset L_{n}^{\prime}$ and $\left(\tilde{L}_{n}\right\rangle$ is decreasing and since $\left\langle L_{n}^{\prime}\right\rangle$ is decreasing and $\lim _{n} L_{n}^{\prime}=\varnothing, \lim \tilde{L}_{n}=\varnothing$. Hence since $\mu \in I_{\sigma}(L)$ by assumption, $\lim _{n} \mu\left(\tilde{L}_{n}\right)=0$. Thus a contradiction has been reached. Therefore the assumption is wrong. Consequently $\nu \in I_{\sigma}\left(L^{\prime}\right)$.

APPLICATION 3.8. Consider any topological sjace $X$ such that $x$ is normal. Then since $F$ is normal, according to Theorem 3.7, the following statement is true: For every element of $I_{\sigma}(F), \mu$, for every element of $I_{R}(F), \nu$, such that $\mu \leq \nu$ on $F, v \in I_{\sigma}(U)$.

COROLLARY 3.9. If $L$ is countably paracompact and normal, then for every element of $I_{\sigma}(L), \mu$, for every element of $I_{R}(L), \nu$, such that $\mu \leq \nu$ on $L, v \in I_{R}^{\sigma}(L)$.

PROOF. Assume $L$ is countably paracompact and normal. Consider any element of $I_{\sigma}(L), \mu$, and any element of $I_{R}(L), \nu$, such that $\mu \leq \nu$ on $L$. Then since $L$ is normal by assumption, by Theorem 3.7, $v \in I_{\sigma}\left(L^{\prime}\right)$. Further, note since $L$ is countably paracompact by assumption, $I_{\sigma}\left(L^{\prime}\right) \subset I_{\sigma}(L)$. Consequently $\nu \in I_{\sigma}(L)$. Then since $\nu$ is $L$-regular, $\nu \in I_{R}^{\sigma}(L)$. 
COROLLARY 3.10. If $L$ is countably paracompact and normal, then for every element of $I_{\sigma}(L), \mu_{1}$, for every element of $I(L), \mu_{2}$, for every element of $I_{R}(L)$, $v$, such that $\mu_{1} \leq \mu_{2} \leq \nu$ on $L, \mu_{2} \in I_{\sigma}(L)$.

APPLICATION 3.11. Consider any topological space $x$ such that $x$ is countably paracompact and normal. Then since $F$ is countabiy paracompact and normal, according to Corollary 3.9, the following statement is true: For every element of $I_{\sigma}(F), \mu$, for every element of $I_{R}(F), \nu$, such that $\mu \leq \nu$ on $F, \nu \in I_{R}^{\sigma}(F)$.

APPLICATION 3.12. Consider any topological space $X$ such that $X$ is $T_{33^{-}}$Then since $Z$ is countably paracompact and normal, according to Corollary 3.9, the following statement is true: For every element of $I_{\sigma}(Z), \mu$, for every element of $I_{R}(Z), \nu$, such that $\mu \leq \nu$ on $Z, \nu \in I_{R}^{\sigma}(Z)$.

DEFINITION 3.13. Consider any element of $I(L), \mu$, such that $\mu$ has the following property:

For every element of $L, A$, such that $\mu\left(\Lambda^{\prime}\right)=1$, there exists an element of $L$, $B$, such that $B \subset A^{\prime}$ and $\mu^{\prime}(B)=1$.

Note if $\mu \in I_{R}(L)$, then $\mu$ has Property $(*)$. For this reason, $\mu$ is said to be weakly regular and $\{\mu \in I(L) \mid \mu$ has Property $(*)\}$ is denoted by $I_{W}(L)$. Thus $I_{R}(L) \subset I_{W}(L)$.

THEOREM 3.14. If $L$ is normal, then $I_{W}(L) \subset I_{R}(L)$.

PROOF. Assume $L$ is nornal. Now, assume $I_{W}(L) \neq \varnothing$ and consicier any element of $I_{W}(L), \mu$. Then there exists an element of $I_{R}(L), \nu$, such that $\mu \leq \nu$ on $L$. Consider any such $\nu$. Note to show $\mu \in I_{R}(L)$, it suffices to show $\mu=\nu$. Further, note for this, it suffices to show $\mu=\nu$ on $L$. Assume the contrary. Then there exists an element of $L, A$, such that $\mu(A)<\nu(A)$. Consider any such $A$. Then $\mu(A)=0$ and $\nu(A)=1$. Hence $\mu\left(A^{\prime}\right)=1$. Hence since $\mu \in I_{W}(L)$ by assumption, there exists an element of $L, B$, such that $B \subset A^{\prime}$ and $\mu^{\prime}(B)=1$, by the definition of $I_{W}(L)$. Consider any such $B$. Now, note since $\mu \in I\left(L^{\prime}\right)$ and $\nu \in I_{R}(L)$ and $\mu \leq \nu$ on $L$ and $L$ is normal by assumption, $\mu^{\prime}=V^{\prime}$ on $L$ by Theorem 3.4. Further, note since $\nu \in I_{R}(L), \nu=\nu^{\prime}$. Consequently $\mu^{\prime}=\nu$ on $L$. Hence since $\mu^{\prime}(B)=1$, $v(B)=1$. Hence since $B \subset A^{\prime}, V\left(A^{\prime}\right)=1$. Hence $v(A)=0$. Thus a contradiction has been reached. Therefore the assumption is wrong. Consequently $\mu=\nu$ on $L$. Consequently $\mu \in I_{R}(L)$. Thus $I_{W}(L) \subset I_{R}(L)$.

REMARK. The converse is false.

COUNTEREXAMPLE. Consider any set $X$ such that $x$ has at least three elements. Now, consider any two elements of $P(X), A, B$, such that $A \neq \varnothing$ and $B \neq \varnothing, A \cap B=\varnothing$, and $A \cup B \neq X$. Further, consider the lattice $L$ described by $L=\{\varnothing, A, B, A \cup B, X\}$.

Next, consider the prime L-filter $F$ described by $F=\{x\}$, then consider the element of $I(L)$ determined by $F$ and denote it by $\mu$. Further, consider the two L-ultrafilters $G_{1}, G_{2}$, described by $G_{1}=\{\varnothing, A, A \cup B, X\}$ and $G_{2}=\{\varnothing, B, A \cup B, X\}$, then consider the elements of $I_{R}(L)$ determined by $G_{1}, G_{2}$, and denote them by $v_{1}, v_{2}$, respectively. Note $I(L)=\left\{\mu, \nu_{1}, v_{2}\right\}$. Show $I_{W}(L) \subset I_{R}(L)$. Note $\mu \notin I_{R}(L)$. Consequently to show $I_{W}(L) \subset I_{R}(L)$, it suffices to show $\mu \notin I_{W}(L)$. Accordingly, note since $\mu(A \cup B)=0, \mu\left(A^{\prime} \cap B^{\prime}\right)=1$. Further, note the only subset of $A^{\prime} \cap B^{\prime}$ is $\varnothing$ and $\mu^{\prime}(\varnothing)=0$. Hence by the definition of $I_{W}(L)$, $\mu \notin I_{W}(L)$. Consequently $I_{W}(L) \subset I_{R}(L)$. 
Finally, note $L$ is not normal.

Thus $I_{W}(L) \subset I_{R}(L)$ and $L$ is not normal.

An alternative proof of the equivalence of parts, (a) and (c) of Theorem 3.4 will be given, which does not involve an outer measure. This proof will be based on a characterization of normality of $L$ in terms of certain $L$-ultrafilters.

Consider any lattice space $\langle X, L\rangle$. Now, consider any element of $I(L), \mu$. Further, consider $L_{L} \in L \mid$ for every element of $L, A$, such that $\mu(A)=I, I \cap A$ $\neq \varnothing\}$ and denote it by $G_{\mu}$.

LEMMA 3.15. $L$ is normal iff for every element of $I(L), \mu, G_{\mu}$ is an $L$-ultrafilter.

PROOF. (a) Assume $L$ is normal and show for every element of $I(L), \mu, G_{\mu}$ is an L-ultrafilter.

(a) Show $G_{\mu}$ is an L-filter

(i) Note $\varnothing \notin G_{\mu}$.

(ii) Show for every two elements of $G_{\mu}, L_{1}, L_{2}, L_{1} \cap L_{2} \in G_{\mu}$. Consider any two elements of $G_{\mu}, L_{1}, L_{2}$. Assume $L_{1} \cap L_{2} \notin G_{\mu}$. Then by the definition of $G_{\mu}$, there exists an element of $L, A$, such that $\mu(A)=1$ and $\left(L_{1} \cap L_{2}\right) \cap A=\varnothing$. Consider any such $A$. Then $A \subset\left(L_{1} \cap L_{2}\right)^{\prime}=L_{1}^{\prime} \cup L_{2}^{\prime}$. Hence since $L$ is normai by assumption, there exist two elements of $L, A_{1}, A_{2}$, such that $A=A_{1} U A_{2}$ and $A_{1} \subset L_{1}$ anci $A_{2} \subset L_{2}^{\prime} \cdot$ Consider any such $A_{1}, A_{2}$. Note since $L_{i} \in G_{\mu}$ and $L_{i} \cap A_{i}=\varnothing$, by the definition of $G_{\mu}, \mu\left(A_{i}\right)=0(i=1,2)$. Hence since $A=A_{1} \cup A_{2}, \mu(A)=0$. Thus a contradiction has been reached. Therefore the assumption is wrong. Consequently, $I_{1} \cap I_{2} \in G_{\mu}$.

(iii) Note for every element of $G_{\mu}, L$, for every element of $L$, $S$, such that $L \subset s, s \in G_{\mu}$.

Consequently $G_{\mu}$ is an L-filter.

(B) Show $G_{\mu}$ is an L-ultrafilter.

Consider any $L$-filter $H$ such that $H \supset G_{\mu}$ and $H \neq G_{\mu}$. Then there exists an element of $H, H$, such that $H \notin G_{\mu}$. Consider any such $H$. Then by the definition of $G_{\mu}$, there exists an element of $L, A$, such that $\mu(A)=1$ and $H \cap A=\varnothing$. Consi$\operatorname{der}$ any such $A$. Then by the definition of $G_{\mu}, A \in G_{\mu}$. Consequently $A \in H$. Thus $H \in H$ and $A \in H$ and $H$ is a filter. Hence $H \cap A \neq \varnothing$. Thus a contradiction has been reached. Therefore the assumption is wrong. Consequently $G_{\mu}$ is an L-ultrafilter.

OBSERVATION. Consider the element of $I_{R}(L)$ determined by $G_{\mu}$ and denote it by $v$. Note $\mu \leq v$ on $L$.

(b) Assume for every element of $I(L), \mu, G_{\mu}$ is an $L$-ultrafilter and show $L$ is normal. For this, use Lemma 3.3, namely, consider any element of $I(L), \mu$, and any two elements of $I_{R}(L), v_{1}, v_{2}$, such that $\mu \leq v_{1}, v_{2}$ on $L$ and show $v_{1}=v_{2}$. Note since $\mu \leq \nu_{1}, v_{2}$ on $L, G_{v_{1}}, G_{v_{2}} \subset G_{\mu}$ by the relevant definition. Hence since $G_{v_{1}}$ and $G_{v_{2}}$ are L-ultrafilters and $G_{\mu}$ is an L-filter by the assumption, $G_{v_{1}}, G_{v_{2}}$ $=G_{\mu}$. Hence $G_{\nu_{1}}=G_{v_{2}}$. Further, note since $G_{\nu_{i}} \supset\left\{A \in L \mid \nu_{i}(A)=1\right\}$ and $\left\{A \in L \mid v_{i}(A)=1\right\}$ is an L-ultrafilter since $v_{i} \in I_{R}(L)$ and $G_{v_{i}}$ is an $L$-filter, $\left\{A \in L \mid v_{i}(A)=1\right\}=G_{v_{i}}(i=1,2)$. Hence since $G_{v_{1}}=G_{v_{2}},\left\{A \in L \mid v_{1}(A)=1\right\}$ 
$=\left\{A \in L \mid v_{2}(A)=1\right\}$. Thus $\nu_{1}=\nu_{2}$ on $L$. Hence $\nu_{1}=v_{2}$. Consequent $1 y$ is normal.

The alternative proof of the equivalence of parts, (a) and (c) of Theorem 3.4 is now given.

(Recall statements (a) and (c) of Theorem 3.4:

(a) $L$ is normal. (c) For every element of $I_{R}\left(L^{\prime}\right), \mu$, for every element of $I_{R}(L), \nu$, such that $\mu \leq \nu$ on $L$, for every element of $L, A$, such that $\nu\left(A^{\prime}\right)=1$, there exists an element of $L, B$, such that $B \subset A^{\prime}$ and $\mu(B)=1_{.}$)

(i) Assume (a) and show (c). Consider any element of $I_{R}\left(L^{\prime}\right), \mu$, any element of $I_{R}(L), \nu$, such that $\mu \leq \nu$ on $L$, and any element of $L, A$, such that $\nu\left(A^{\prime}\right)=1$. Show there exists an element of $L, B$, such that $B \subset A^{\prime}$ and $\mu(B)=1$. Note $\mu \in I(L)$. Consider $G_{\mu}$. Note $G_{\mu}=\{L \in L \mid$ for every element of $L$, $A$, such that $\mu(A)=1, L \cap A \neq \varnothing\}$ by definition. Further, note since $L$ is normal by assumption, by (Lemma 3.15, (a)), $G_{\mu}$ is an L-ultrafilter. Consider the element of $I_{R}(L)$ determined by $G_{\mu}$ and denote it by $\rho$. Note $\mu \leq \rho$ on $L$. Thus $\mu \in I(L)$ and $\nu, \rho \in I_{R}(L)$ and $\mu \leq \nu, \rho$ on $L$. Hence since $L$ is normal, $\nu=\rho$. Then since $\nu\left(A^{\prime}\right)=1, \rho\left(A^{\prime}\right)=1$. Hence $\rho(A)=0$. Therefore $A \notin G_{\mu}$ (Property of $\rho_{\bullet}$ ) Hence by the definition of $G_{\mu}$, there exists an element of $L, B$, such that $\mu(B)=1$ and $A \cap B=\varnothing$. Consider any such $B$. Then $B \in L$ and $B C A^{\prime}$ and $\mu(B)=1$.

(ii) Assume (c) and show (a). For this, assume the contrary. Then there exist two elements of $L, A, B$, such that $A \cap B=\varnothing$ and for every two elements of $L, C, D$, such that $C^{\prime} \supset A$ and $D^{\prime} \supset B, C^{\prime} \cap D^{\prime} \neq \varnothing$. Consider any such $A, B$. Now, consider $\left\{L^{\prime} \in L^{\prime} \mid L^{\prime}\right\lrcorner A$ or $\left.L^{\prime} \supset B\right\}$ and denote it by $E$. Note $A \neq \varnothing$ and $B \neq \varnothing$. Consequently $E$ has the Finite Intersection Property. Hence there exists an element of $I_{R}\left(L^{\prime}\right), \mu$, such that for every element of $E, L^{\prime}, \mu\left(L^{\prime}\right)=1$. Consider any such

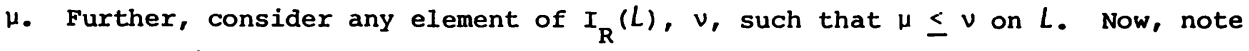
since $\nu \in I(L)$ and $A \cap B=\varnothing, \nu(A)=0$ or $\nu(B)=0$. Assume $\nu(A)=0$ (without loss of generality). Then $\nu\left(A^{\prime}\right)=1$. Thus $\mu \in I_{R}\left(L^{\prime}\right), v \in I_{R}(L)$ and $\mu \leq \nu$ on $L$, and $A \in L$ and $\nu\left(A^{\prime}\right)=1$. Hence since (c) is true by assumption, there exists an element of $L, C$, such that $C \subset A^{\prime}$ and $\mu(C)=1$. Consider any such $C$. Then $C^{\prime} \in E$ and $\mu\left(C^{\prime}\right)=0$. Thus a contradiction has been reached. Theréfore the assumption is wrong. Consequently $L$ is normal.

4. LATTICE NORMALITY AND COUNTABLY SUBADDITIVE OUTER MEASURE.

In this section we work with an arbitrary set $x$ and an arbitrary lattice on $x$,

L. We introduce a certain countably subadditive outer measure on $P(X)$ and use it to obtain conditions for $L$ to be normal.

DEFINITION 4.1. Consider any lattice space $(X, L)$. Now, consider any element of $M(L), \mu$, and the function $\mu^{\prime \prime}$ on $P(X)$ determined by $\mu "(A)=\inf \left\{\Sigma_{k=1}^{\infty} \mu\left(L_{k}^{\prime}\right) \mid\right.$ $L_{k} \in L$ for every $k$ and $\left.U_{k} L_{k}^{\prime} \supset A\right\}$.

PROPOSITION 4.2 . (i) $\mu^{\prime \prime}$ is a countably subadditive outer measure.

(ii) $\mu^{\prime \prime} \leq \mu^{\prime}$.

(iii) If $\mu \in I(L)$, then $\mu^{\prime \prime}(P(x)) \subset\{0,1\}$ 。

(Proof omitted.)

PROPOSITION 4.3. (i) If $\mu \in I_{\sigma}(L)$, then $\mu \leq \mu$ " on $L$.

(ii) If $\mu \in I(L)$ and $\mu(x)=\mu^{\prime \prime}(X)$, then $\mu \in I_{\sigma}(L)$. 
PROOF. (i) Assume $\mu \in I_{v}(L)$. To show $\mu \leq \mu$ " on $L$, assume the contrary. Then there exists an element of $L, A$, such that $\mu^{\prime \prime}(A)<\mu(A)$. Consider any such A. Then since $\mu^{\prime \prime}(A)=\inf \left\{\sum_{k=1}^{\infty} \mu\left(L_{k}^{\prime}\right) \mid L_{k} \in L\right.$,for every $k$ and $\left.\sum_{k} L_{k}^{\prime} \supset A\right\}$ by the definition of $\mu$ ", there exists a sequence in $L,\left(L_{k}\right)$, such that $U_{k} L_{k}^{\prime} \supset A$ and $\sum_{k} \mu\left(L_{k}^{\prime}\right)<\mu(A)$. Consider any such $\left(L_{k}\right)$. Then since $\mu \in I(L), \mu(A)=1$ and for every $k, \mu\left(L_{k}\right)=0$. Consequently $\cap_{k}\left(A \cap L_{k}\right)=\varnothing$ and for every $k$, $\mu\left(A \cap I_{k}\right)=1$. Now, for every natural number $n$, consider $\eta_{k=1}^{n}\left(A \cap L_{k}\right)$. Note $\eta_{k=1}^{n}\left(A \cap L_{k}\right) \in L_{i}$ set $n_{k=1}^{n}\left(A \cap L_{k}\right)=\hat{L}_{n} ;$ note $\mu\left(\hat{L}_{n}\right)=1$. Consider $\left(\hat{L}_{n}\right)$. Note $\left\langle\hat{L}_{n}\right\rangle$ is in $L$ and $\left\langle\hat{L}_{n}\right\rangle$ is decreasing and $\lim _{n} \hat{L}_{n}=n_{n} \hat{L}_{n}=n_{k}\left(A \cap L_{k}\right)=\varnothing$. Hence since $\mu \in I_{\sigma}(L)$ by assumption, $\lim _{n} \mu\left(\hat{L}_{n}\right)=0$. Thus a contradiction has been reached. Therefore the assumption is wrong. Consequently $\mu \leq \mu$ " on $L$.

(ii) (Proof omitted.)

NOTATION. Consider any lattice space $(x, L)$. Now, consider any element of $I(L), \mu$, such that $\mu$ has the following property:

For every sequence in $L,\left(L_{n}\right)$, such that $n_{n} L_{n} \in L, \mu\left(n_{n} L_{n}\right)=\inf \left(\mu\left(L_{n}\right)\right.$; $\mathrm{n} \in \mathrm{N}\} . \quad(* *)$

Note if $\mu \in I^{\sigma}(L)$, then $\mu$ has Property $(* *)$ and if $\mu$ has Property $(* \star)$, then $\mu \in I_{\sigma}(L)$. Thus $I^{\sigma}(L) \subset\{\mu \in I(L) \mid \mu$ has Property $(* *)\} \subset I_{\sigma}(L)$. Set $\{\mu \in I(L)$ $\{\mu$ has Property $(* *)\}=J(L)$. Thus $I^{\sigma}(L) \subset J(L) \subset I_{\sigma}(L)$.

PROPOSITION 4.4. If $\mu \in I(L)$, then $\mu=\mu^{\prime \prime}$ on $L^{\prime}$ iff $\mu \in J(L)$.

PROOF. Assume $\mu \in I(L)$.

(i) Assume $\mu=\mu "$ on $L^{\prime}$ and show $\mu \in J(L)$. Assume the contrary. Then by the relevant definition, there exists a sequence in $\left.L, i L_{n}\right)$, such that $n_{n} L_{n} \in L$ and $\mu\left(n_{n} L_{n}\right) \neq \inf \left\{\mu\left(L_{n}\right) ; n \in N\right\}$. Consider any such $\left(L_{n}\right\rangle$. Now, note since $\left(L_{n}\right\rangle$ is in $L$ and $U_{n} L_{n}^{\prime} \supset U_{n} L_{n}^{\prime}$, by the definition of $\mu^{\prime \prime}, \mu^{\prime \prime}\left(U_{n}^{\prime} L_{n}^{\prime}\right) \leq \sum_{n} \mu\left(L_{n}^{\prime}\right)$. Now, note since $\mu\left(n_{n} L_{n}\right) \neq \inf \left\{\mu\left(L_{n}\right) ; n \in N\right\}, \mu\left(n_{n} L_{n}\right)<\inf \left\{\mu\left(L_{n}\right) ; n \in N\right\}$. Hence since $\mu \in I(L), \mu\left(n_{n} L_{n}\right)=0$ and for every $n, \mu\left(L_{n}\right)=1$. Hence for every $n, \mu\left(L_{n}^{\prime}\right)=0$. Consequently $\mu^{\prime \prime}\left(U_{n} L_{n}^{\prime}\right)=0$. Further, note since $\mu=\mu$ " on $L^{\prime}$ by assumption and $U_{n} L_{n}^{\prime} \in L^{\prime}$ because $n_{n} L_{n} \in L, \mu\left(U_{n} L_{n}^{\prime}\right)=\mu$ " $\left(U_{n} L_{n}^{\prime}\right)$. Consequently $\mu\left(U_{n} L_{n}^{\prime}\right)=0$. Hence $\mu\left(n_{n} L_{n}\right)=1$. Thus a contradiction has been reached. Therefore the assumption is wrong. Consequently $\mu \in J(L)$.

(ii) (Proof omitted.)

PROPOSITION 4.5. If $L$ is complement generated, then $J(L) \subset I_{w}(L)$. PROOF. Assume $L$ is complement generated. Note since $I^{\sigma}(L) \subset J(L), J(L) \neq \varnothing$. Consider any element of $J(L), \mu$. To show $\mu \in I_{W}(L)$, use the relevant definition, namely, consider any element of $L, L$, such that $\left.\mu^{\prime} L^{\prime}\right)=1$ and show there exists an element of $L, \tilde{L}$, such that $\tilde{L} \subset L^{\prime}$ and $\mu^{\prime}(\tilde{L})=1$. Note since $L \in L$ and $L$ is complement generated by assumption, there exists a sequence in $L,\left(\hat{L}_{k}\right)$, such that $L=\eta_{k} \hat{L}_{k}^{\prime} \cdot$ Consider any $\operatorname{such}\left\langle\hat{L}_{k}\right\rangle$. Then $L^{\prime}=U_{k} \hat{L}_{k}$. Further, note since $\mu \in J(L)$ by assumption, by Proposition 4.4, $\mu=\mu^{\prime \prime}$ on $L^{\prime}$. Consequently $1=\mu\left(L^{\prime}\right)=\mu^{\prime \prime}\left(L^{\prime}\right)$ $=\mu^{\prime \prime}\left(U_{k} \hat{L}_{k}\right) \leq \sum_{k} \mu "\left(\hat{L}_{k}\right)$. Hence there exists a value of $k, m$, such that $\mu^{\prime \prime}\left(\hat{L}_{m}\right)$ $=1$. Consider any such $m$. Then since $\mu^{\prime \prime} \leq \mu^{\prime}, \mu^{\prime}\left(\hat{\mathrm{L}}_{\mathrm{m}}\right)=1$. Thus $\hat{\mathrm{L}}_{\mathrm{m}} \in L$ and $\hat{L}_{m} \subset L^{\prime}$ and $\mu^{\prime}\left(\hat{L}_{m}\right)=1$. Consequently $\mu \in I_{W}(L)$. Thus $J(L) \subset I_{W}(L)$. 'PHEOREM 4.6. If $L$ is normal and complement generated, then $J(L)=I_{R}^{\sigma}(L)$. 
PROOF. Assume $L$ is normal and complement generated.

(a) Show $J(L) \subset I_{R}^{\sigma}(L)$. Note since $L$ is complement generated, by proposition 4.5, $J(L) \subset I_{W}(L)$. Further, note since $L$ is normal, by Theorem 3.14, $I_{W}(L) \subset I_{R}(L)$. Consequently $J(L) \subset I_{R}(L)$. Hence since $J(L) \subset I_{\sigma}(L), J(L) \subset I_{R}^{\sigma}(L)$.

(B) Show $I_{R}^{\sigma}(L) \subset J(L)$. Note $I_{R}^{\sigma}(L) \subset I^{\sigma}(L) \subset J(L)$.

$(\gamma)$ Consequently $J(L)=I_{R}^{\sigma}(L)$.

APPLICATION 4.7. Consider any topological space $x$ such that $x$ is perfectly normal. Then since $F$ is normal and complement generated by definition, by Theorem $4.6, J(F)=I_{R}^{\sigma}(F)$.

APPLICATION 4.8. Consider any topological space $X$ such that $X$ is $T_{3 \frac{1}{2}}$. Then since $Z$ is normal and complement gencratcd, by Theorem 4.6,J(Z) $=I_{R}^{\sigma}(Z)$.

THEOREM 4.9. If $L$ is normal and countably paracompact and $\mu \in I_{\sigma}(L)$, then $\mu^{\prime \prime}=\mu^{\prime}$ on $L$.

PROOF. Assume $L$ is normal and countably paracompact and $\mu \in I_{\sigma}(L)$. Note since $\mu \in I(L)$ by assumption, there exists an element of $I_{R}(L), \nu$, such that $\mu \leq \nu$ on $L$. Consider any such $\nu_{\text {. Thus }} \mu \in I\left(L^{\prime}\right)$ and $\nu \in I_{R}(L)$ and $\mu \leq \nu$ on $L$. Hence since $L$ is normal by assumption, by Theorem $3.4, \mu^{\prime}=V^{\prime}$ on $L$. Now, note since $L$ is countably paracompact and normal by assumption and $\mu \in I_{\sigma}(L)$ and $\nu \in I_{R}(L)$ and $\mu \leq \nu$ on $L$, by corollary 3.9, $\nu \in I_{\sigma}(L)$. Further, note [since $\nu \in I_{\sigma}(L)$, by (Proposition 4.3, (ii), $v \leq v^{\prime \prime}$ on $L$ ] and $v^{\prime \prime} \leq v^{\prime}$ and since $v$ is $L$-regular, $v^{\prime}=v^{\prime}$ Hence $\nu^{\prime}=v^{\prime \prime}$ on $L$. Also, note since $\mu \leq \nu$ on $L, v^{\prime \prime} \leq \mu^{\prime \prime}$. Consequently $\nu^{\prime} \leq \mu^{\prime \prime}$ on $L$. Then since $\mu^{\prime \prime} \leq \mu^{\prime}, \nu^{\prime} \leq \mu^{\prime} \leq \nu^{\prime}$ on $L$. Hence since $\mu^{\prime}=\nu^{\prime}$ on $L, \mu^{\prime \prime}=\mu^{\prime}$ on $L$.

APPLICATION 4.10. Consider any topological space $X$ such that $X$ is $T_{3 \frac{1}{2}}$ Then since $Z$ is normal and countably paracompact, according to Theorem 4.9, the following statement is true: If $\mu \in I_{\sigma}(Z)$, then $\mu^{\prime \prime}=\mu^{\prime}$ on $Z$.

APPLICATION 4.11. Consider any topological space $\mathrm{X}$ such that $\mathrm{X}$ is $\mathrm{T}_{1}$ and o-dimensional. Then since $C$ is normal and countably paracompact, according to Theorem 4.9, the following statement is true: If $\mu \in I_{\sigma}(C)$, then $\mu "=\mu^{\prime}$ on $C$.

THEOREM 4.12. If $L$ is normal and $\delta$ and $\mu \in I_{\sigma}(L)$, then $\mu^{\prime \prime}=\mu^{\prime}$ on $L$.

PROOF. Assume $L$ is normal and $\delta$ and $\mu \in I_{\sigma}(L)$. Note to show $\mu^{\prime \prime}=\mu^{\prime}$ on $L$, since $\mu^{\prime \prime} \leq \mu^{\prime}$, it suffices to show for every element of $L, L, \mu^{\prime \prime}(L) \not \mu^{\prime}(L)$. Assume the contrary. Then there exists an element of $L, A$, such that $\mu^{\prime \prime}(A)<\mu^{\prime}(A)$. Consider any such $A$. Then $\mu^{\prime \prime}(A)=0$ and $\mu^{\prime}(A)=1$. Now, note since $\mu^{\prime \prime}(A)=0$, there exists a sequence in $L_{,}\left\langle\hat{L}_{k}\right\rangle$, such that $U_{k} \hat{L}_{k}^{\prime} \supset A$ and $\sum_{k} \mu\left(\hat{L}_{k}^{\prime}\right)=0$. Consider any such $\left\langle\hat{\bar{L}}_{k}\right\rangle$. Note $A \subset U_{k} \hat{L}_{k}^{\prime}=\left(\Omega_{k} \hat{L}_{k}\right)^{\prime}$ and since $L$ is $\delta$ by assumption, $n_{k} \hat{L}_{k} \in L_{\text {. }}$ set $\cap_{k} \hat{L}_{k}=B$. Then $A \subset B^{\prime}$. Now, use the assumption that $L$ is normal and $\mu \in I_{\sigma}(L)$ to show $\mu^{\prime}(A)=0$, thus reaching a contradiction.

APPLICATION 4.13. Consider any topological space $X$ such that $X$ is normal. Then since $F$ is normal and $\delta$, according to Theorem 4.12, the following statement is true: If $\mu \in I_{\sigma}(F)$, then $\mu "=\mu$ ' on $F$.

APPLICATION 4.14. Consider any topological space $x$ such that $X$ is $T_{3 \frac{1}{2}}$ Then since $Z$ is normal and $\delta$, according to Theorem 4.12, the following statement is true: If $\mu \in I_{\sigma}(Z)$, then $\mu^{\prime \prime}=\mu^{\prime}$ on $Z$. 
ACKNOWLEDGEMENT. The author wishes to express his aprreciation to Long Island University for partial support of the present work through a grant of released time from teaching dutics.

\section{REFERENCES}

1. ALEXANDROFF, A. D., Additive set-functions in abstract spaces, Mat. sb. (N.S.) 9 (51) (1941), 563-628.

2. BACHMAN, G. and SULTAN, A., Regular lattice measures: mappings and spaces, Pacific J. Math. 67, no. 2, (1976), 291-326.

3. On regular extensions of measures, Pacific J. Math. 86, no. 2, $(1980), 389-395$.

4. CAMACHO, J., On maximal measures with respect to a lattice, Internat. J. Math. \& Math. Sci., 14, No. 1 (1991), 93-98.

5. EID, G., On normal lattices and Wallman spaces, Internat. J. Math. \& Math. Sci. $13(1)(1990), 31-38$.

6. FROLIK, Z., Prime filters with the C.I.P., Comm. Math. Univ. Carolinae, 13, (1972), 553-575.

7. NÖBELING, G., Grundlagen der analytischen Topologie, Springer, Berlin, 1954.

8. SZETO, M., Measure repeletness and mapping preservations, J. Indian Math. Soc. 43 (1979), 35-52. 


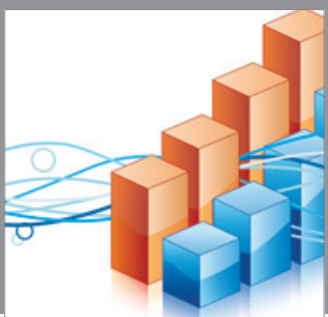

Advances in

Operations Research

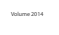

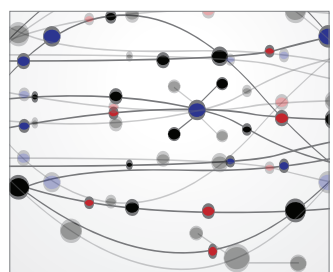

\section{The Scientific} World Journal
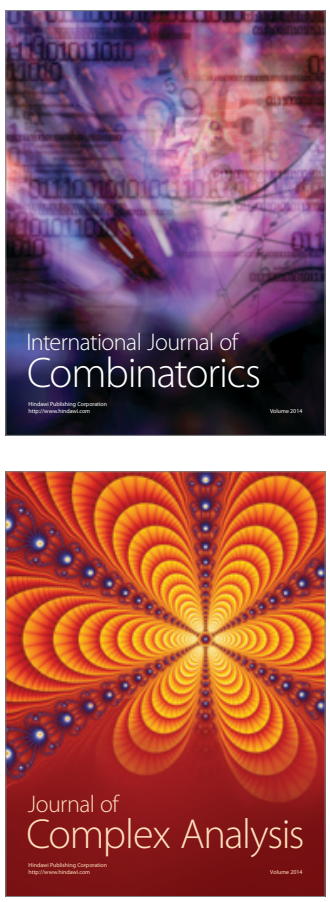

International Journal of

Mathematics and

Mathematical

Sciences
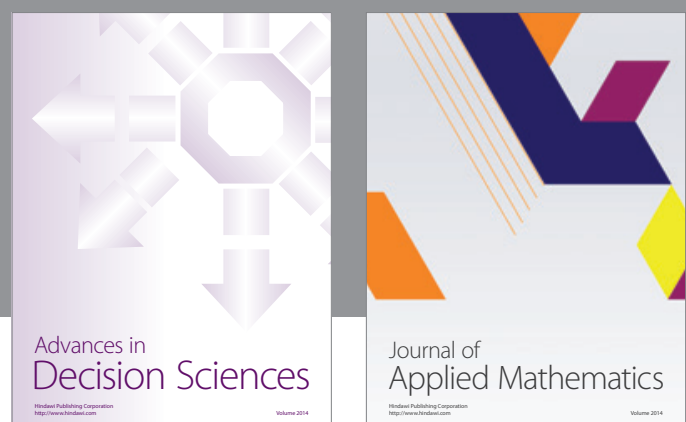

Journal of

Applied Mathematics
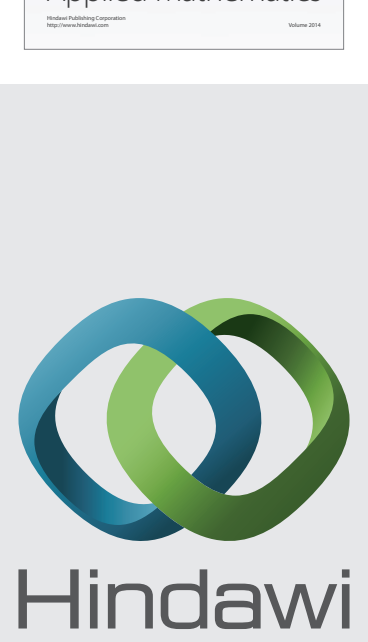

Submit your manuscripts at http://www.hindawi.com
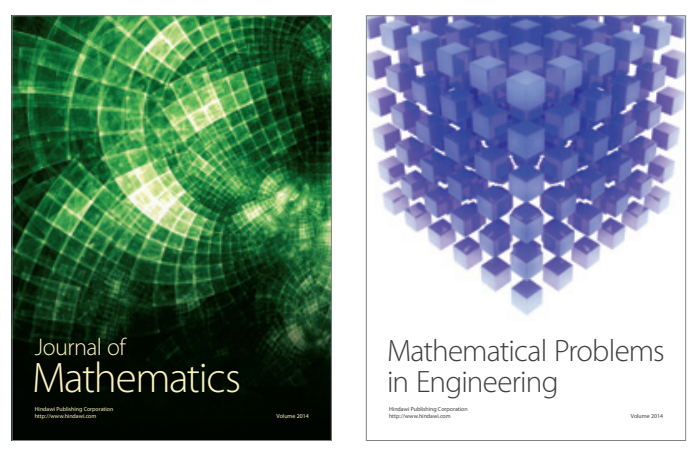

Mathematical Problems in Engineering
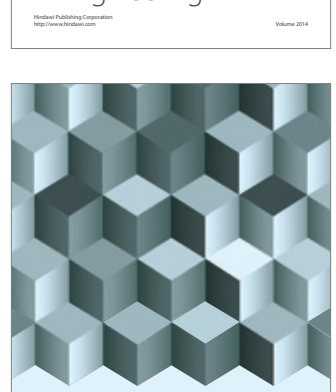

Journal of

Function Spaces
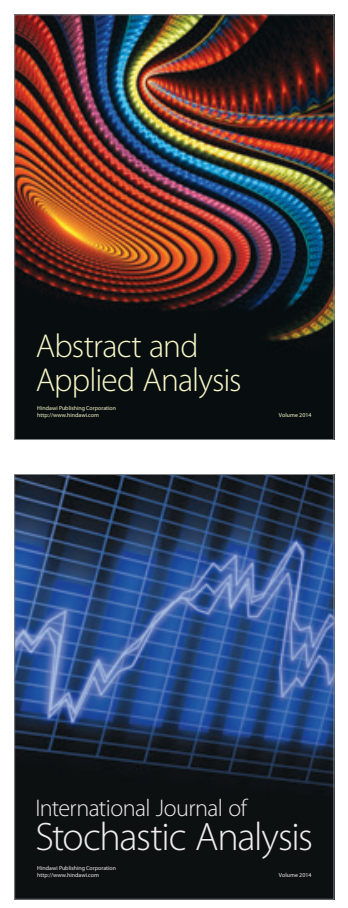

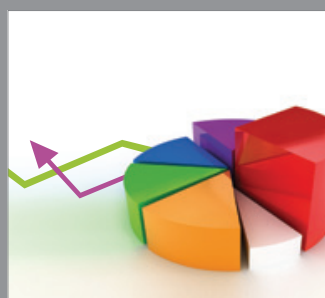

ournal of

Probability and Statistics

Promensencen
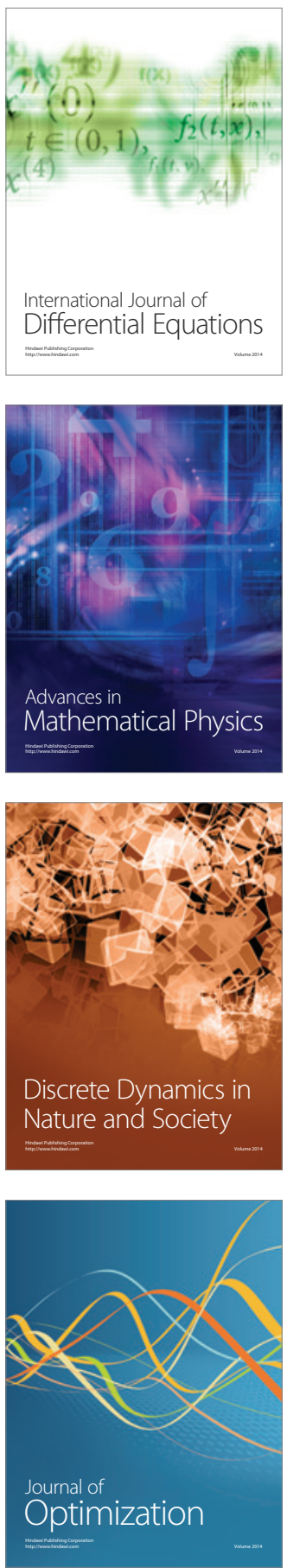